\title{
Nature of synchronization transitions in random networks of coupled oscillators
}

\author{
Jaegon Um, ${ }^{1}$ Hyunsuk Hong, ${ }^{2}$ and Hyunggyu Park ${ }^{1}$ \\ ${ }^{1}$ School of Physics, Korea Institute for Advanced Study, Seoul 130-722, Korea \\ ${ }^{2}$ Department of Physics and Research Institute of Physics and Chemistry, \\ Chonbuk National University, Jeonju 561-756, Korea
}

(Dated: June 25, 2018)

\begin{abstract}
We consider a system of phase oscillators with random intrinsic frequencies coupled through sparse random networks, and investigate how the connectivity disorder affects the nature of collective synchronization transitions. Various distribution types of intrinsic frequencies are considered: uniform, unimodal, and bimodal distribution. We employ a heterogeneous mean-field approximation based on the annealed networks and also perform numerical simulations on the quenched Erdös-Rényi networks. We find that the connectivity disorder drastically changes the nature of the synchronization transitions. In particular, the quenched randomness completely wipes away the diversity of the transition nature and only a continuous transition appears with the same mean-field exponent for all types of frequency distributions. The physical origin of this unexpected result is discussed.
\end{abstract}

PACS numbers: 64.60.aq, 05.70.Fh, 05.45.Xt

In recent years, there has been an explosion of research on the critical phenomena in complex networks [1]. Most studies of various systems in complex networks so far have been accomplished by means of the heterogenous mean-field (MF) theory. The heterogeneous MF theory is based on the annealed networks where the links are not fixed but fluctuate in time, i.e., at each time step, the neighbors of a node are chosen randomly with its given degree. However, the connectivity in many real systems is indeed quenched one, i.e., the links are fixed permanently in time once they are formed. Nevertheless, as the critical phenomena in networks are assumed to belong to some kind of MF universality classes, it may be natural to believe that fluctuations induced by the quenched connectivity disorder are irrelevant in describing the MF-type critical phenomena except a finite shift of the critical threshold. In fact, many cases such as the Ising model and the contact process have proven to be applicable except intriguing finite-size effects [2 44]. The synchronization problem with an unimodal distribution of random frequencies also belongs to the case [5-12].

Very recently, there is a claim in the epidemic spreading model via the so-called quenched MF analysis 13. that the quenched disorder affects the phase transition considerably and completely wipes away the transition predicted by the annealed MF theory. There, the unboundedness of degrees in networks is crucial to make the critical threshold to vanish in the thermodynamic limit. However, the validity of the quenched MF theory is controversial because it still ignores dynamic (temporal) fluctuations which tend to make the endemic phase unstable [14 16].

In this work, we present an example where the quenched connectivity disorder changes its phase transition nature. We consider a system of coupled phase oscillators in random networks and pay attention to its collective synchronization behavior. In particular, we take into account various distribution types of random intrinsic frequencies and analyze the system by means of the annealed MF theory and also extensive numerical simulations on both quenched and annealed networks. We find that the connectivity disorder preserves the synchronization transition at a finite (but shifted) threshold, but sometimes with completely different transition nature. For example, a discontinuous transition becomes continuous in the presence of the connectivity disorder. We argue that this surprising result is due to a substantial change in the effective random frequency distribution caused by the quenched connectivity.

We begin with a finite population of $N$ coupled phase oscillators on the Erdös-Rényi (ER) random network [17]. To each vertex $j$ of the network, we associate an oscillator whose state is described by the phase angle $\phi_{j}$ governed by

$$
\dot{\phi}_{j}=\omega_{j}-J \sum_{l=1}^{N} a_{j l} \sin \left(\phi_{j}-\phi_{l}\right),
$$

where $\omega_{j}$ represents the intrinsic frequency of the $j$ th oscillator, chosen from a given distribution $g(\omega)$. In this work, we restrict our discussion to the symmetric distribution $g(\omega)=g(-\omega)$. The coupling constant $J$ denotes the attractive coupling $(J>0)$, so the neighboring oscillators favor their phase difference minimized. The $\left\{a_{j l}\right\}$ is the adjacency matrix with its elements given by $a_{j l}=a_{l j}=1$ when the vertices $j$ and $l$ are connected (linked), and $a_{j l}=0$ otherwise. The degree of the vertex $i$ is defined as the number of linked vertices; $k_{i}=\sum_{j} a_{i j}$. The links are fully independent of each other, then the degree distribution $P(k)$ for the ER networks is given by the Poisson distribution [17],

$$
P(k)=\frac{\langle k\rangle^{k} e^{-\langle k\rangle}}{k !}
$$

where $\langle k\rangle$ is the mean degree given by $\langle k\rangle=\sum_{k} P(k) k$.

We explore the collective synchronization using the MF theory. Let us first introduce a set of local ordering fields 
defined by

$$
H_{j} e^{i \theta_{j}} \equiv \sum_{l} a_{j l} e^{i \phi_{l}}
$$

where $H_{j}$ and $\theta_{j}$ denote the amplitude and the mean phase of the local field at vertex $j$, respectively. With this local field, Eq. (1) is rewritten as

$$
\dot{\phi}_{j}=\omega_{j}-J H_{j} \sin \left(\phi_{j}-\theta_{j}\right) .
$$

Following the previous studies [5-8], we assume that the link-to-link fluctuation in the local fields is negligible under the random connection: If the network is well connected with no fragmentation into local communities, it is expected that a cluster of the entrained oscillators will influence on the dynamics of all oscillators through a global ordering field $H e^{i \theta}$. This MF assumption allows us to replace the term $e^{i \phi_{l}}$ in the sum of Eq. (3) with the global field $H e^{i \theta}$ acting through the edge connecting vertices $j$ and $l$. As all edges contribute the same, the local field is simply given by the degree $k_{j}$ times the global field. This is the key MF approximation taken in [5 8]. In fact, this MF procedure is identical to the MF theory on the annealed networks where the adjacency matrix is approximately given by the connecting probability as

$$
a_{j l} \approx \frac{k_{j} k_{l}}{N\langle k\rangle}
$$

In this scheme, the global field can be explicitly given as

$$
H e^{i \theta} \approx k_{j} H_{j} e^{i \theta_{j}} \approx \frac{1}{N} \sum_{l} \frac{k_{l}}{\langle k\rangle} e^{i \phi_{l}}
$$

Substituting the local fields by the global field approximately in Eq. (4), the annealed MF equation is given by

$$
\dot{\phi}_{j}=\omega_{j}-k_{j} J H \sin \left(\phi_{j}-\theta\right) .
$$

Note that the oscillator at vertex $j$ feels the effective coupling constant $k_{j} J$ with the global field $H$. The selfconsistency equation for $H$, Eq. (6), then reads [8]

$$
H=\frac{1}{N} \sum_{j=1}^{N} \frac{k_{j}}{\langle k\rangle} \sqrt{1-\left(\frac{\omega_{j}}{k_{j} J H}\right)^{2}} \Theta\left(1-\frac{\left|\omega_{j}\right|}{k_{j} J H}\right),
$$

where $\Theta(x)$ is the Heaviside step function: $\Theta(x)=1$ for $x \geq 0$ and 0 otherwise. This implies that only the $e n-$ trained (synchronized in frequency) oscillators that have the intrinsic frequencies $\omega_{j}$ restricted by $\left|\omega_{j}\right|<k_{j} J H$ contribute to the global field $H$. Note that the connectivity disorder is reduced to the degree fluctuation only, in this annealed MF description. In the continuum limit of $N \rightarrow \infty, \mathrm{Eq}$. (8) can be rewritten as [8]

$$
H=\frac{1}{\langle k\rangle} \sum_{k} P(k) k U(k J H),
$$

where

$$
U(x)=\int_{-x}^{x} d \omega g(\omega) \sqrt{1-(\omega / x)^{2}}
$$

For comparison, we revisit the globally interacting oscillator system on a complete graph $(\mathrm{CG})$ where $a_{j l}=1$ for all pairs of $(j, l)$ and $P(k)=\delta_{k, N}$ with the coupling constant rescaled as $J \rightarrow J / N$. In this case, the above MF procedure is exact except dynamic fluctuations, which yields

$$
\dot{\phi}_{j}=\omega_{j}-J H \sin \left(\phi_{j}-\theta\right) \text { and } H=U(J H) .
$$

In heterogeneous networks compared to the above $\mathrm{CG}$ case, oscillators with higher degree $k$ tend to behave with a stronger effective coupling constant $k J$, thus become entrained earlier at smaller $J$. Therefore, oscillators with the same intrinsic frequency $\omega$ will be entrained in the order of degree $k$ from above. However, as the oscillator frequencies are also distributed randomly, there may be an intricate interplay between the frequency and the degree distribution.

We first focus on a simple and interesting case that the intrinsic frequencies are drawn randomly from a uniform (flat) and bounded distribution given by

$$
g(\omega)= \begin{cases}\frac{1}{2 b}, & |\omega| \leq b \\ 0, & \text { otherwise }\end{cases}
$$

The function $U(x)$ in Eq. (10) then leads to

$$
U(x)= \begin{cases}\frac{1}{2 b} \int_{-x}^{x} d \omega \sqrt{1-(\omega / x)^{2}}=\frac{\pi}{4 b} x & \text { for } x \leq b \\ \frac{1}{2 b} \int_{-b}^{b} d \omega \sqrt{1-(\omega / x)^{2}} & \text { for } x>b\end{cases}
$$

For the globally interacting oscillators on the CG, it is easy to show from Eqs. (11) and (13) that there is a jump of the order parameter $H$ with jump size $\Delta H=\pi / 4$ at $J=J_{c}=4 b / \pi[18,19]$. The reason for a finite jump is trivial mathematically, because there is no nonzero solution for $H$ possible when $x=J H \leq b$. The firstorder transition nature could be understood intuitively as follows: For the case of a unimodal frequency distribution $\left(g^{\prime \prime}(0)<0\right)$, oscillators with small frequencies $\omega \approx 0$ can start to be entrained in frequency for a sufficiently strong coupling constant $J_{c}$, and the frequency entrainment and phase synchronization spreads over to oscillators with higher $\omega$ continuously with increasing $J>J_{c}$. This synchronization mechanism leads to a continuous transition. However, with a broader distribution $\left(g^{\prime \prime}(0) \geq 0\right)$, abundance of high-frequency oscillators hinders and thus destabilizes the attempted entrainment of small-frequency oscillators through direct interactions (links) between them. A flat distribution is a limiting case where all oscillators wait to be entrained until the highest-frequency ones $(\omega= \pm b)$ become stabilized. Then, all oscillators suddenly become entrained together in frequency (though their phases are still not fully ordered), which drives the first-order discontinuous 
synchronization transition. In the case of a bimodal distribution with exponentially decaying tails, not all but still a finite fraction of oscillators with frequencies in between two bimodal peaks will be entrained together all of a sudden. Thus, again, a discontinuous transition is expected. Therefore, the transition nature on the CG is quite sensitive to the characteristics of the frequency distribution function $g(\omega)$, in particular, its curvature property at the entrainment frequency. Note that the entrainment frequency is zero due to the symmetric property of $g(\omega)$.

Now we return to the ER network with the uniform frequency distribution. From Eqs. (9) and (13), it is straightforward to derive

$$
H=\frac{\pi J H}{4 b\langle k\rangle}\left[\left\langle k^{2}\right\rangle-\sum_{k>b / J H} k^{2} P(k) F\left(\frac{b}{k J H}\right)\right],
$$

where

$$
F(x)=1-\frac{2}{\pi}\left[\sin ^{-1} x+x \sqrt{1-x^{2}}\right],
$$

where $F(x)$ is positive for $0 \leq x<1$. By a simple analysis, we find the incoherent solution, $H=0$ for $J<J_{c}=\frac{4 b\langle k\rangle}{\pi\left\langle k^{2}\right\rangle}$ and a partially coherent solution (nonzero $H$ ) for $J>J_{c}$.

Near $J \gtrsim J_{c}$, the global field value $H$ can be evaluated by solving the equation

$$
\epsilon \equiv \frac{J}{J_{c}}-1 \approx \frac{1}{\left\langle k^{2}\right\rangle} \sum_{k>b / J_{c} H} k^{2} P(k) F\left(\frac{b}{k J_{c} H}\right),
$$

where $\epsilon$ is the reduced coupling constant. For small $H$, the summation is only over high $k$, where $P(k)$ decays exponentially fast with $k$ for the ER network, see Eq. (2). Thus, only a few terms of $k \gtrsim b /\left(J_{c} H\right)$ are dominant in the summation. Using the expansion of $F(x)$ near $x \lesssim 1$, the above equation becomes

$$
\epsilon \sim \frac{1}{\left\langle k^{2}\right\rangle} \sqrt{\frac{b}{J_{c} H}} P\left(\frac{b}{J_{c} H}\right),
$$

which leads to the logarithmic scaling as

$$
H \approx \frac{b}{J_{c}}|\ln \epsilon|^{-1} .
$$

Note that there is a continuous transition at $J=J_{c}$, even though the logarithmic scaling implies a very steep increase of $H$ near $J \gtrsim J_{c}$. This can be contrasted to the CG case where the discontinuous transition is found with a big jump at $J=J_{c}$. Nevertheless, this is not quite surprising because oscillators at vertices with many links in the tail part of $P(k)$ feel strong effective interactions $(\sim k J)$ as discussed before, so become entrained much easily even for high-frequency oscillators. Therefore, we expect a hierarchical synchronization (entrainment) in the order of the degree $k$ from above. If the degree is unbounded with an exponentially vanishing population, $P(k) \sim e^{-c k}$ for large $k$, the same logarithmic scaling is expected by Eq. (17). In the case of the CG case, a similar hierarchical synchronization is also found for a continuous transition with a unimodal frequency distribution, but in the order of the intrinsic frequency $|\omega|$ from below. It is interesting to note that the amplitude of the logarithmic scaling, $b / J_{c}=\frac{\pi\left\langle k^{2}\right\rangle}{4\langle k\rangle}$, depends only on the network property and not on the width $b$ of the frequency distribution.

If we consider a random network with a finite upper bound for the degree $\left(k \leq k_{m}\right)$, the effective interaction is also bounded. So we expect that oscillators even with the highest degree $k_{m}$ should wait until the highest-frequency oscillators with $\omega= \pm b$ become stabilized, similar to the CG case. Thus, this leads to a discontinuous transition at $J_{c}=\frac{4 b\langle k\rangle}{\pi\left\langle k^{2}\right\rangle}$ with jump $\Delta H=b /\left(J_{c} k_{m}\right)$, which can be easily derived from Eq. (14). In the limit of infinite $k_{m}$, the jump vanishes and a continuous transition is recovered. As an example, in the case of the regular random network with $P(k)=\delta_{k, k_{0}}$, we get a discontinuous synchronization transition at $J_{c}=\frac{4 b}{\pi k_{0}}$ with $\Delta H=\pi / 4$.

For a unimodal frequency distribution with $g^{\prime \prime}(0)<0$, it is possible to find a nonzero solution for small $x=k J H$ for sufficiently high $J[8,11]$. It can be easily seen by expanding $g(\omega)$ for small $\omega$, up to $\mathcal{O}\left(\omega^{2}\right)$, in Eq. (10). Due to the symmetry of $g(\omega)$, the self-consistency equation, Eq. (9), carries only odd-power terms in $H$ as

$$
H \simeq \frac{\pi J H}{2\langle k\rangle}\left[g(0)\left\langle k^{2}\right\rangle-\frac{\left\langle k^{4}\right\rangle}{8}\left|g^{\prime \prime}(0)\right|(J H)^{2}+\cdots\right]
$$

The onset of synchronization is given by $J_{c}=\frac{2\langle k\rangle}{\pi g(0)\left\langle k^{2}\right\rangle}$ and the global field $H$ near $J \gtrsim J_{c}$ scales as

$$
H \approx A \epsilon^{1 / 2},
$$

with $A^{2}=\frac{2 \pi^{2} g^{3}(0)}{\left|g^{\prime \prime}(0)\right|} \frac{\left\langle k^{2}\right\rangle^{3}}{\left\langle k^{4}\right\rangle\langle k\rangle^{2}}$. This ordinary MF result is valid for any degree distribution $P(k)$ with finite $\left\langle k^{4}\right\rangle$, regardless of the boundedness of the degree distribution. The cases with diverging $\left\langle k^{4}\right\rangle$ were discussed in details with anomalous finite size scaling in our previous study [8]. With the gaussian $g(\omega)=g_{G}(\omega)=$ $\frac{1}{\sqrt{2 \pi} \sigma} e^{-\omega^{2} / 2 \sigma^{2}}$, the amplitude $A$ becomes independent of the width $\sigma$ of the distribution function, i.e. $A^{2}=$ $\pi \frac{\left\langle k^{2}\right\rangle^{3}}{\left\langle k^{4}\right\rangle\langle k\rangle^{2}}$.

For a binomial distribution with $g^{\prime \prime}(0)>0$, one needs higher-order terms in the expansion of Eq. (19) to find a nonzero solution for $H$. But it is impossible to find a vanishingly small $H$ solution, so a discontinuous transition is expected [18, 20]. In this work, we consider the double gaussian distribution, i.e. $g(\omega)=\frac{1}{2}\left[g_{G}\left(\omega-\omega_{0}\right)+\right.$ $\left.g_{G}\left(\omega+\omega_{0}\right)\right]$ with $\omega_{0}>\sigma$. On the CG, it is known that the so-called standing wave phase appears in between the incoherent and partially synchronized phase [18, 20] when the bimodality becomes stronger (large $\omega_{0} / \sigma$ ). Recently, a more complex dynamic feature was found on the 
CG 21]. It would be interesting to study how this feature may change in the annealed sparse network. In any case, the annealed MF theory with a binomial distribution on the ER network predicts neither the ordinary continuous transition, nor the logarithmic continuous transition, but a discontinuous transition at $J_{c}=\frac{2\langle k\rangle}{\pi g(0)\left\langle k^{2}\right\rangle}$ with the random initial distribution of $\left\{\phi_{j}\right\}$.

In order to see whether all these interesting features found in the annealed MF theory can persist in quenched networks, we perform extensive numerical simulations on the ER networks. The ER networks are generated for $\langle k\rangle=4$ up to the system size $N=64000$. Using Heun's method, we integrate Eq. (11) numerically with a discrete time step $\delta t=0.01$ up to $t=10^{3}$. Initial values for $\left\{\phi_{j}\right\}$ are chosen randomly and the data are collected and averaged from $t=500$. We also average the data over $100-500$ realizations of networks and intrinsic frequency distributions for each network size. We also perform numerical integrations in the annealed ER networks, where the adjacency matrix $a_{i j}$ is replaced by Eq. (5).

We measure the phase synchronization order parameter in the steady state, defined as [18]

$$
\Delta=\frac{1}{N}\left|\sum_{j=1}^{N} e^{i \phi_{j}}\right|
$$

which can be rewritten in the annealed MF theory as [8]

$$
\Delta=\frac{1}{N} \sum_{j=1}^{N} \sqrt{1-\left(\frac{\omega_{j}}{k_{j} J H}\right)^{2}} \Theta\left(1-\frac{\left|\omega_{j}\right|}{k_{j} J H}\right) .
$$

Comparing Eq. (21) to the global field $H$ in Eq. (6), it is easy to see that $\Delta$ is proportional to $H$ for small $H$. In the annealed network, one can easily derive the relation as $\Delta \approx \frac{\pi}{2} g(0)\langle k\rangle J_{c} H$ for small $H$ from Eq. (22). Therefore, the scaling behavior should be identical for $\Delta$ and $H$ near the continuous transition and the discontinuity in $H$, if any, should also appear in $\Delta$.

First, we take a uniform frequency distribution with $b=1 / 2$ in Eq. (12). Figure 1 shows the behavior of the order parameter $\Delta$ as a function of the coupling strength $J$ for (a) the annealed ER network and (b) the quenched one. For the annealed networks, a very steep increase of the order parameter is found for large $N$ near the exact $J_{c}=2 /(5 \pi) \approx 0.1273$, which is consistent with the logarithmic scaling in Eq. (18). In fact, by solving Eq. (14) for $H$ in the $N=\infty$ limit and evaluating $\Delta$ of Eq. (22), the dashed curve is drawn in Fig. 1(a), which serves well as the asymptotic limit.

In contrast, for the quenched networks in Fig. 1(b), the order parameter increases rather smoothly near $J_{c} \approx$ $0.165(5)$. To investigate the transition nature more precisely, we utilize the standard finite-size-scaling (FSS) theory as

$$
\Delta N^{\beta / \bar{\nu}}=f\left(\epsilon N^{1 / \bar{\nu}}\right)
$$
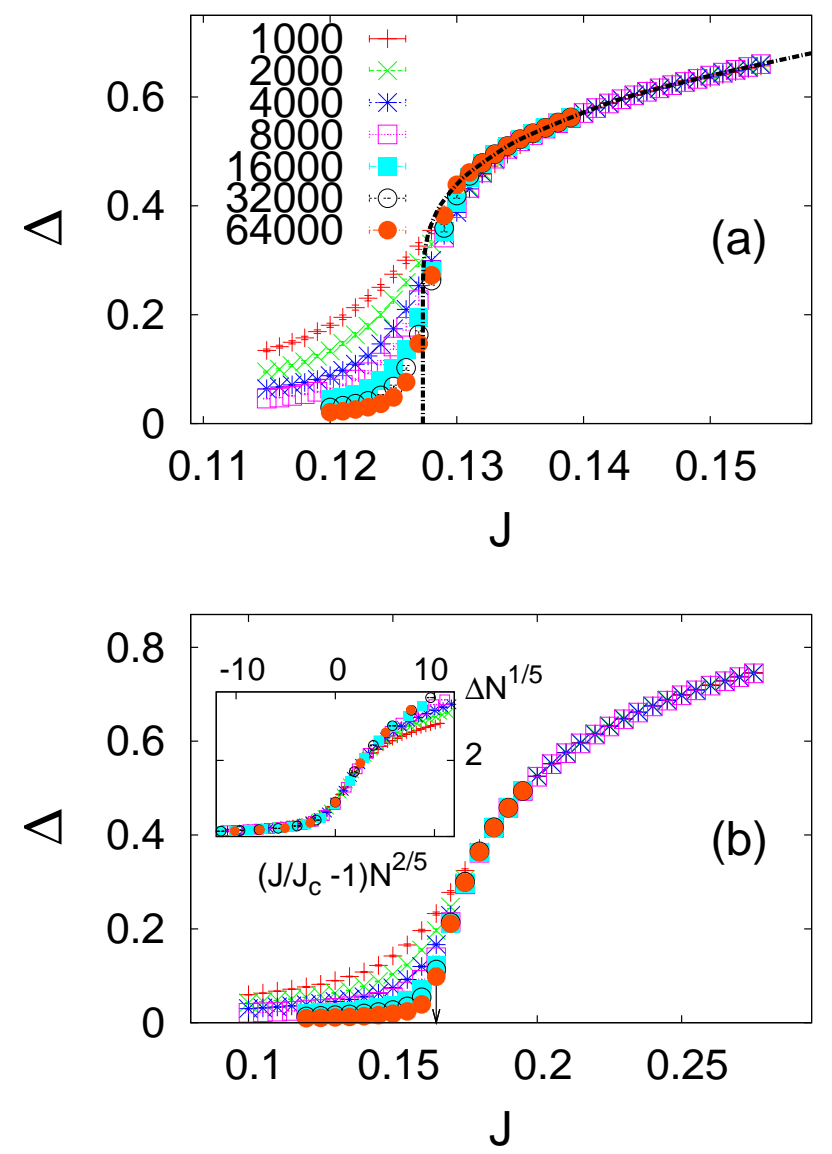

FIG. 1: (Color online) The order parameter $\Delta$ versus $J$ for the uniform frequency distribution with $b=1 / 2$ in (a) the annealed ER networks and (b) the quenched ER networks with various sizes $N$. The dashed curve in (a) indicates the logarithmic scaling near $J_{c}=2 /(5 \pi) \approx 0.1273$ predicted by the annealed MF theory in the $N=\infty$ limit. Inset of (b) shows the collapse of all finite-size data on the scaling curve $f(x)$ with $\beta / \bar{\nu}=1 / 5$ and $\bar{\nu}=5 / 2$ with $J_{c}=0.165(5)$ as in Eq. (24).

where the scaling function $f(x)$ is given by

$$
f(x) \sim \begin{cases}x^{\beta} & x \gg 0, \\ \text { const. } & x=0, \\ (-x)^{-(\bar{\nu} / 2-\beta)} & x \ll 0,\end{cases}
$$

with the order parameter exponent $\beta$ and the FSS exponent $\bar{\nu}$. This scaling behavior yields $\Delta \sim \epsilon^{\beta}$ for $\epsilon>0$ in the limit of large $N, \Delta \sim N^{-\beta / \bar{\nu}}$ at $\epsilon=0$, and $\Delta \sim N^{-1 / 2}$ for $\epsilon<0$ for large $N$. The inset of Fig. 1(b) shows an excellent agreement with the FSS with

$$
\beta=1 / 2 \quad \text { and } \quad \bar{\nu}=5 / 2 .
$$

This indicates that the synchronization transition belongs to the ordinary MF universality class with $\beta=1 / 2$. Moreover, the FSS exponent value of $\bar{\nu}=5 / 2$ agrees with the analytic result of the CG case with a unimodal 

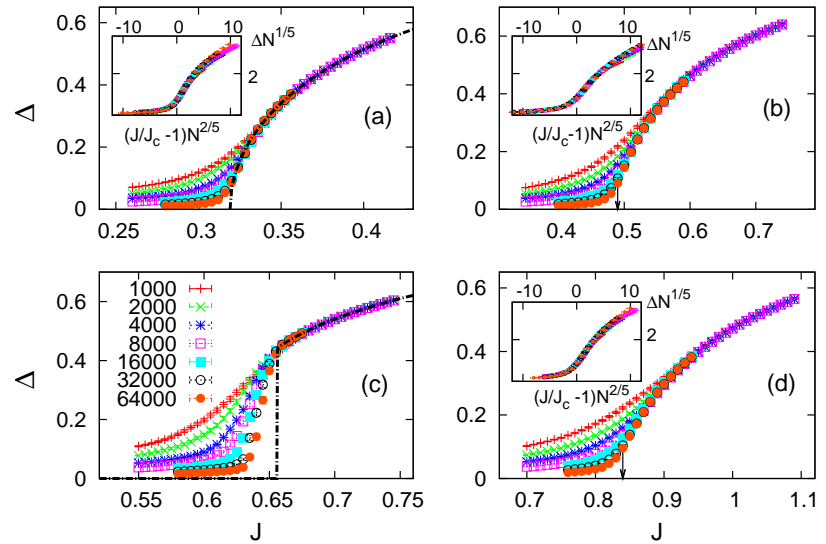

FIG. 2: (Color online) The order parameter $\Delta$ versus $J$ for the gaussian (unimodal) frequency distribution with $\sigma=1$ in (a) the annealed ER networks and (b) the quenched ER networks, and for the double gaussian (bimodal) distribution with $\sigma=1$ and $\omega_{0}=1.2$ in (c) the annealed ER networks and (d) the quenched ER networks with various sizes $N$. The dashed curves in (a) and (c) indicate the annealed MF prediction in the $N=\infty$ limit. Insets of (a), (b), and (d) show the collapse of all finite-size data on the scaling curve $f(x)$ with $\beta / \bar{\nu}=1 / 5$ and $\bar{\nu}=5 / 2$ with $J_{c}=2 \sqrt{2} /(5 \sqrt{\pi}) \approx 0.319,0.49(1)$, and $0.84(1)$, respectively, as in Eq. (24). In (c), as predicted from the annealed MF theory, the finite-size data seem to indicate the discontinuous transition at $J_{c}=2 \sqrt{2} /(5 \sqrt{\pi}) * e^{0.72} \approx$ 0.656 with random initial conditions.

distribution with frequency fluctuations 22, 23]. Thus, quite surprisingly, for the uniform distribution, the synchronization transition nature changes from a discontinuous to a logarithmic and finally to the ordinary MF continuous transition, as the underlying network topology changes from the CG to the annealed and finally to the quenched ER networks. We have also performed the numerical integrations in the quenched regular random network with $k_{0}=4$, and found the same ordinary MF transition (not shown here). This implies that all distinctive features in the transition nature are washed away when the quenched disorder in connectivity is introduced.

Similar to the uniform distribution, we have performed the numerical integrations for the gaussian (unimodal) frequency distribution with $\sigma=1$ and the double gaussian (bimodal) one with $\sigma=1$ and $\omega_{0}=1.2$, both in the annealed ER network and in the quenched network. As expected, Fig. 2(a) and (b) show that the case with the unimodal distribution exhibits the ordinary MF continuous transition in both networks [11]. Also, Fig. 2(c) confirms the discontinuous transition predicted by the annealed MF theory. However, surprisingly again, the case with the bimodal distribution exhibits the simple MF transition with $\beta=1 / 2$ and $\bar{\nu}=5 / 2$ in the quenched network, as seen in Fig. 2(d). Moreover, there is no indication of the presence of any dynamic
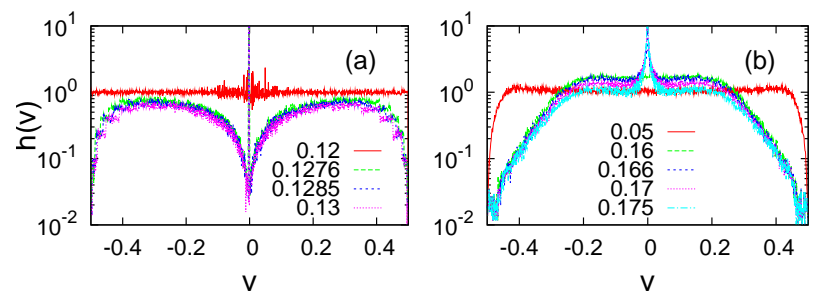

FIG. 3: (Color online) Histogram $h(v)$ of the mean angular velocity $v$ of oscillators in the steady state for the uniform frequency distribution with $b=1 / 2$ in (a) the annealed ER network and (b) the quenched network. The data are obtained from one sample simulation of each network of big size $N=$ 512000 . Note that $J_{c} \approx 0.1273$, and 0.165 , respectively, for each network.

phase including the standing wave phase. Hence, the quenched disorder fluctuations in network connectivity seem to wipe out all interesting features found in the $\mathrm{CG}$, and drives all synchronization transitions into the ordinary MF universality class. We also examined the extreme bimodal case with only two symmetric frequencies allowed, i.e. $g(\omega)=\left[\delta\left(\omega-\omega_{0}\right)+\delta\left(\omega+\omega_{0}\right)\right] / 2$, and found the same ordinary MF synchronization transition in the quenched ER networks (not shown here).

Summarizing our numerical results, the ordinary MF synchronization transition with $\beta=1 / 2$ and $\bar{\nu}=5 / 2$ is found in the quenched ER and regular networks, regardless of the intrinsic frequency distribution function $g(\omega)$. This is quite remarkable, because the transition nature crucially depends on the shape of $g(\omega)$ in the annealed networks and also in the CG. It obviously raises a question how the quenched connectivity disorder affects the transition nature, against the conventional wisdom that quenched disorder fluctuations in the MF regime are irrelevant in terms of the universality. It is also noteworthy to mention that the scaling function $f(x)$ in the ordinary MF universality class is not universal by itself, i.e. varies with the frequency distribution $g(\omega)$ and the underlying network structure.

Now we explore the role of the quenched connectivity disorder in synchronization. First, we measure the mean angular velocity of the $j$ th oscillator in the steady state, defined by

$$
v_{j}=\frac{\phi_{j}\left(t_{f}+t_{i}\right)-\phi_{j}\left(t_{i}\right)}{t_{f}}
$$

with the initial measurement time $t_{i}=500$ to reach the steady state and the large duration time $t_{f}=10000$ for a good numerical precision. Then, we establish the normalized histogram $h(v)$ from the mean velocity data $\left\{v_{j}\right\}$ of all $N$ oscillators, which is shown in Fig. 3 at various values $J$ with the uniform frequency distribution with $b=1 / 2$. The numerical precision of $v$ to establish $h(v)$ is given by $\Delta v=10^{-3}$.

In the annealed ER network as well as in the CG, it is straightforward to show analytically that the mean os- 
cillator velocity $v_{j}$ does not change from its intrinsic frequency $\omega_{j}$ in the incoherent phase $\left(J<J_{c} \approx 0.1273\right)$, so its distribution $h(v)$ also remains unchanged from the intrinsic frequency distribution $g(\omega)$. Going into the partially synchronized phase for $J>J_{c}$, a sharp $\delta$-function starts to develop at $v=0$, which implies the emergence of the macroscopic entrainment of oscillators with the zero entrainment velocity, see Fig. 3(a). Depletion of $h(v)$ near $v=0$ reveals that most of entrained oscillators near $J \gtrsim J_{c}$ originate from those with small intrinsic frequencies $\omega$.

On the other hand, in the quenched ER network, the histogram $h(v)$ deviates from $g(\omega)$ considerably even in the incoherent phase $\left(J<J_{c} \approx 0.165\right)$, which indicates that the quenched connectivity causes the significant modification of the oscillator velocity even before the macroscopic entrainment emerges, see Fig. 3(b). In particular, high-frequency oscillators become quite slowed down and the fraction of small-frequency oscillators increases before the transition. However, the velocity distribution $h(v)$ seems still flat near $v=0$, thus this observation by itself can not explain why the ordinary MF continuous transition should appear in the quenched network. For $J \gtrsim J_{c}$, one can see a slow increase of the entrained oscillator peak at $v=0$, which is consistent with the ordinary MF transition.

One important ingredient missing in the above study is the distinct local environment surrounding oscillators such as the number of links (degree) and the velocities of neighboring oscillators. For example, if an oscillator with small $\omega$ is linked to neighboring oscillators with large positive $\omega$ only, it is very difficult to stabilize this oscillator due to the hindrance of neighboring ones. In contrast, some oscillators with large $\omega$ can join the entrainment rather easily if the neighboring oscillators try to cancel out their velocity $\omega$ in the opposite direction. Moreover, abundance of high-frequency oscillators could not destabilize all low-frequency oscillators because of the limited quenched connections between them.

In order to see the above mechanism from the numerical simulations, we also measure the histogram $h(\omega)$ of the intrinsic frequency $\omega$ for the entrained oscillators at various values of $J$, see Fig. (4 We again take the uniform frequency distribution with $b=1 / 2$. As the integration of $h(\omega)$ represents the fraction of the entrained oscillators, it is smaller than 1 for any finite $J$. In fact, it should vanish in the $N=\infty$ limit for $J<J_{c}$.

In the annealed network, no oscillators with nonzero $\omega$ can be entrained for $J<J_{c}$, while oscillators with $|\omega| \leq k J H$ become entrained for $J>J_{c}$, which produces a step-like pattern in $h(\omega)$ due to the discreteness of the degree distribution. As there exists a bound of the intrinsic frequency $(|\omega| \leq b)$, all-frequency oscillators (especially including high-frequency ones) with highenough connectivity $k$ get entrained simultaneously near the transition. As we integrate $h(\omega)$ to get the entrainment fraction for $J \gtrsim J_{c}$, the significant change as a function of $J$ occurs for large $\omega$, which again implies that
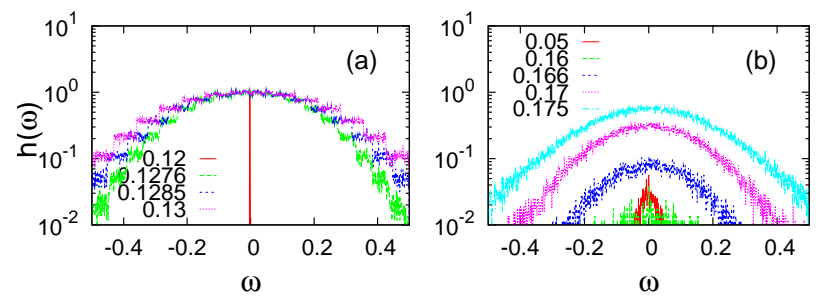

FIG. 4: (Color online) Histogram $h(\omega)$ of the intrinsic frequency $\omega$ of the entrained oscillators for the uniform frequency distribution with $b=1 / 2$ in (a) the annealed ER network and (b) the quenched network. The histogram is normalized by $N$, so its integration represents the fraction of the entrained oscillators. The data are obtained from one sample simulation of each network of big size $N=512000$. Note that $J_{c} \approx 0.1273$, and 0.165 , respectively, for each network.

the tail part of the degree distribution $P(k)$ plays a dominant role on the critical behavior near the transition.

In the quenched network, the situation is completely different, see Fig. 4(b). Some oscillators even with $\omega=0$ cannot remain entrained due to interactions with neighboring oscillators. For $J<J_{c}$, the entrainment fraction is very small around $\omega=0$ and vanishes in the $N=\infty$ limit. This vanishingly small entrainment originates from local clusters of slow oscillators with small $\omega$ in the favorable environment (for example, intrinsic frequencies of neighboring oscillators are small and balanced). As $J$ approaches $J_{c}$ from below, these locally entrained clusters grow in size by inviting neighboring oscillators with small $\omega$ hierarchically and also merge together. Then, finally macroscopically entrained clusters appear as $J$ crosses over $J_{c}$. As can be seen in Fig. 4(b), the major contribution to the entrainment comes from slow oscillators. As $J$ increases, slow oscillators in a less favorable environment join the entrainment as well as faster oscillators in a favorable environment. Therefore, the original shape of $g(\omega)$, whether it is flat or bimodal, does not play an important role near the transition. Furthermore, the shape of $h(\omega)$ near the transition is unimodal near $\omega=0$, as slower oscillators contribute more to the entrainment. This unimodality leads to the ordinary MF continuous transition as in the annealed networks and also in the CG with a unimodal frequency distribution $g(\omega)$.

We can devise a simple model incorporating the quenched environment quantitatively as follows. Neighboring oscillators in the environment affect the given oscillator by effectively modifying its intrinsic frequency, even before the transition. We assume that the modification is additive and proportional to the average intrinsic frequency of neighbors, well inside of the incoherent phase. Then, the effective intrinsic frequency $\omega_{j}^{e}$ of the $j$ th oscillator may be written as

$$
\omega_{j}^{e}=\omega_{j}+\eta_{j}
$$


with

$$
\eta_{j} \simeq \frac{\alpha(J)}{k_{j}} \sum_{l} a_{j l} \omega_{l}
$$

where $\alpha(J)$ is an unknown (increasing) function of $J$ with $\alpha(0)=0$.

For simplicity, we also assume that $\left\{\eta_{j}\right\}$ are independent each other (not true if two oscillators share the same neighbor). Then, $\left\langle\eta_{j} \eta_{l}\right\rangle=0$ for $j \neq l$. We can also get $\left\langle\eta_{j}\right\rangle=0$ and $\left\langle\eta_{j}^{2 n}\right\rangle=\alpha^{2 n}\left\langle\omega^{2 n}\right\rangle / k_{j}^{2 n-1}$ for $n=1,2, \ldots$ As the moments decrease rapidly with $n$ for $k_{j}>1$, we ignore the moments for $n \geq 3$ and assume the distribution of $\eta_{j}$ is gaussian, i.e. $\tilde{g}\left(\eta_{j}\right)=\frac{1}{\sqrt{2 \pi} \tilde{\sigma}_{j}} e^{-\eta_{j}^{2} / 2 \tilde{\sigma}_{j}^{2}}$ with $\tilde{\sigma}_{j}^{2}=\alpha^{2}\left\langle\omega^{2}\right\rangle / k_{j}$. Note that the distribution is sharper for large $k_{j}$.

With this distribution $\tilde{g}\left(\eta_{j}\right)$, the effective frequency distribution of the oscillator $j$ is given as

$$
\begin{aligned}
\bar{g}\left(\omega_{j}^{e}\right) & =\iint d \omega d \eta_{j} \tilde{g}\left(\eta_{j}\right) g(\omega) \delta\left(\omega_{j}^{e}-\omega-\eta_{j}\right) \\
& =\frac{1}{\sqrt{2 \pi} \tilde{\sigma}_{j}} \int d \omega e^{-\left(\omega_{j}^{e}-\omega\right)^{2} / 2 \tilde{\sigma}_{j}^{2}} g(\omega) .
\end{aligned}
$$

The additional concaveness in the frequency distribution near $\omega_{j}^{e}=0$ is generated due to the environmental modification $\eta_{j}$. The curvature $\bar{g}^{\prime \prime}(0)$ at the entrainment frequency is

$$
\bar{g}^{\prime \prime}(0)=\frac{1}{\sqrt{2 \pi} \tilde{\sigma}_{j}^{3}} \int d \omega\left(\frac{\omega^{2}}{\tilde{\sigma}_{j}^{2}}-1\right) e^{-\omega^{2} / 2 \tilde{\sigma}_{j}^{2}} g(\omega),
$$

which can be negative with large $\tilde{\sigma}_{j}$ (low degree $k_{j}$ or large $\alpha$ ) for any distribution shape of $g(\omega)$. And this effective frequency distribution is realized well before the macroscopic entrainment begins. So the entrainment (synchronization) mechanism operates on the basis of the effective frequency distribution, instead of the original intrinsic frequency distribution. Hence, the unimodality of the effective frequency distributions of oscillators with low degrees would be the underlying reason why the ordinary MF universality is found for all types of $g(\omega)$.

In order to check our scenario of the effective frequency distribution, we plot the mean velocity (effective frequency) versus the average intrinsic frequencies of neighboring oscillators. The mean velocity is measured for oscillators with $\omega_{j} \approx 0$ in numerical simulations with the uniform distribution of $g(\omega)$ with $b=1 / 2$ on the regular random networks with $k_{0}=4$. In Fig. 5 the data at $J=0.72 J_{c}$ seem to be consistent with our scenario with the linear slope $\alpha \approx 0.45$. We also collected data at various different values of $J<J_{c}$, all of which can be fitted well with a straight line. Their linear slopes are plotted in Fig. 6 for the unimodal, uniform, and bimodal distributions of $g(\omega)$. As expected, $\alpha(J)$ increases at the beginning and saturates as $J$ increases. However, it starts to decrease slightly around $70 \%$ of $J / J_{c}$, which may be due to the presence of local mesoscopic entrained

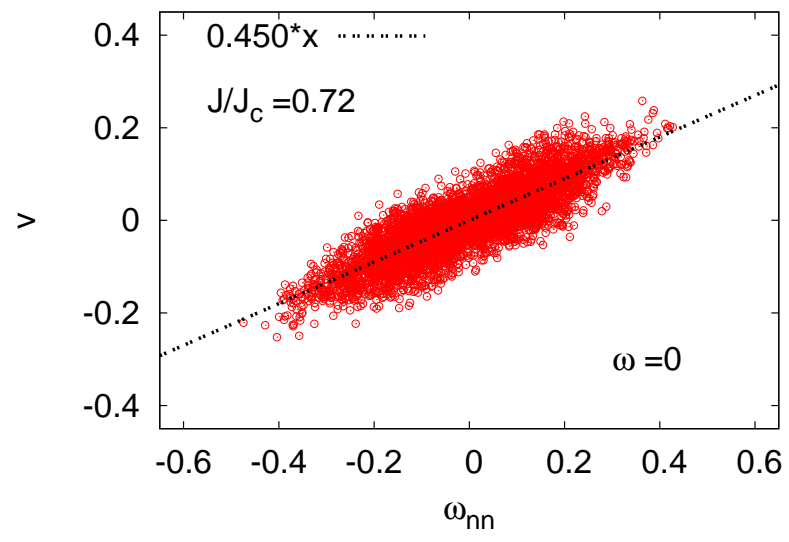

FIG. 5: (Color online) Mean velocity (effective frequency) $v$ of the $j$ th oscillator versus the average intrinsic frequencies of neighboring oscillators $\omega_{n n}=\sum_{l} a_{j l} \omega_{l} / k_{j}$. The mean velocity is averaged for oscillators with $\omega_{j} \approx 0$ in the incoherent phase, in numerical simulations on the regular random networks of size $N=256000$ with $k_{0}=4$ with the uniform distribution with $b=1 / 2$. The dashed line is the straight line fitting the data in average.

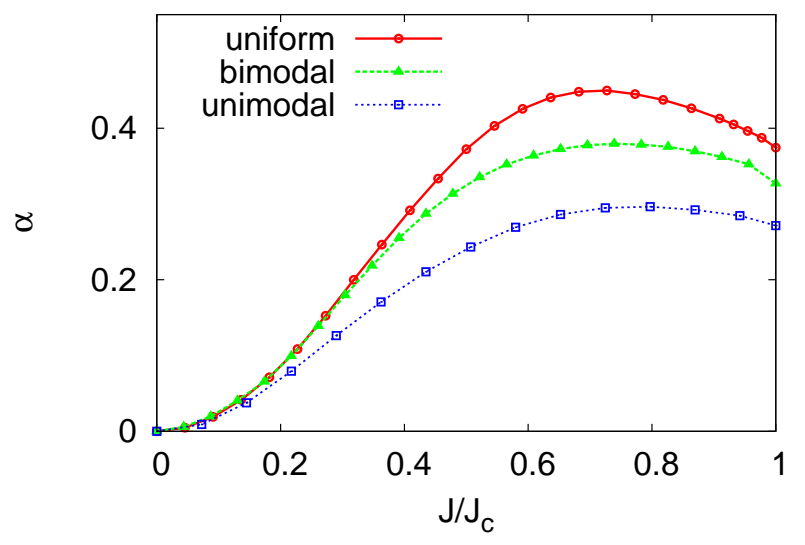

FIG. 6: (Color online) Linear slope $\alpha$ versus $J / J_{c}$ for various distribution types of the intrinsic frequency $g(\omega)$.

clusters. Near $J / J_{c} \lesssim 1, \alpha$ is still fairly finite, so the effective frequency distribution should operate well as the basis for the emergence of macroscopic entrained clusters.

Even though the proposed simple mechanism describes qualitatively how the ordinary MF transition emerges in quenched networks, the quantitative prediction is still quite away from the numerical data. For example, the amplitude of the order parameter, $A$ in Eq. (20), substituting the effective distribution $\bar{g}(\omega)$, turns out to be a few times larger than what is obtained by numerical simulations. Moreover, when the bimodality becomes bigger, the effective curvature of Eq. (30) can remain positive, so the continuous transition is not predicted with this simple mechanism. However, the continuous transition 


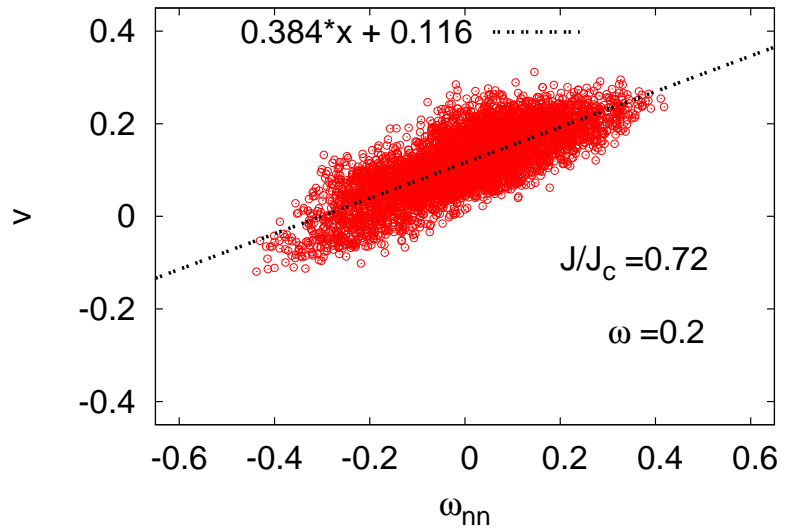

FIG. 7: (Color online) Mean velocity (effective frequency) $v$ of the $j$ th oscillator versus the average intrinsic frequencies of neighboring oscillators $\omega_{n n}=\sum_{l} a_{j l} \omega_{l} / k_{j}$. The mean velocity is averaged for oscillators with $\omega_{j} \approx 0.2$ in the incoherent phase, in numerical simulations on the regular random networks of size $N=256000$ with $k_{0}=4$ with the uniform distribution with $b=1 / 2$. The dashed line is the straight line fitting the data in average.

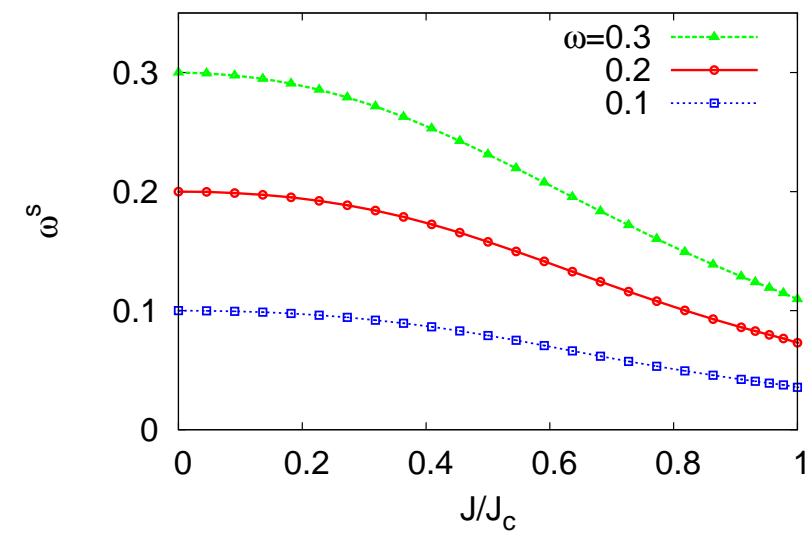

FIG. 8: (Color online) Shifted average velocity $\omega^{s}$ versus $J / J_{c}$ for various intrinsic frequency $\omega$ 's with the uniform distribution of the intrinsic frequency $g(\omega)$.

is found in numerical simulations. In the extreme bimodal case with only two symmetric frequencies $\left( \pm \omega_{0}\right)$, one can easily show that the curvature is always positive for any $k_{j}$ with $\alpha<1$ and the measured value of $\alpha(J)$ in simulations seems to be always less than 1 as seen in Fig. 6.

Therefore, there should be a secondary mechanism to fill this discrepancy. In fact, we find a tendency for oscillators with nonzero $\omega_{j}$ to attain a smaller frequency in average due to the interactions with neighboring oscillators. In Fig. 7 the mean velocity of oscillators with $\omega_{j} \approx 0.2$ is plotted against the average intrinsic frequencies of neighboring oscillators. It shows a nice straight line but with a shifted average velocity $\omega_{j}^{s} \approx 0.116$. With this observation, we modify our simple mechanism in Eq. (27) as

$$
\omega_{j}^{e}=\omega_{j}^{s}\left(\omega_{j} ; J\right)+\eta_{j}
$$

where $\omega_{j}^{s}\left(\omega_{j} ; J\right)$ is the shifted average velocity with $\omega_{j}^{s}\left(\omega_{j} ; 0\right)=\omega_{j}$. We plot the average shift velocity $\omega^{s}(J)$ in Fig. 8 for the uniform distribution of $g(\omega)$. For different types of $g(\omega)$, a similar behavior is found. One can notice that the shift is quite sizable as $J$ approaches $J_{c}$, especially for large $\omega$. This strong shift towards a smaller frequency should strengthen the unimodality of the effective frequency distribution $\bar{g}\left(\omega^{e}\right)$, which can explain the finding of the ordinary MF continuous transition even for the extreme bimodal case in quenched networks.

In summary, we have investigated the synchronization transition of the random frequency oscillators coupled through sparse random networks. In particular, we considered various different shapes of intrinsic frequency distributions in the ER networks, and analyzed the system by means of the annealed MF theory. The annealed MF theory predicts distinctive transition nature depending on the curvature shape of the intrinsic frequency distribution. However the numerical simulations in the quenched network show the ordinary MF continuous transition with the same critical exponents, regardless of the frequency distribution shape. This implies that the quenched connectivity drastically changes the nature of the synchronization transitions. We discuss the underlying physical origin for this remarkable result and provide various evidences how the quenched disorder changes effectively the frequency distribution in the incoherent phase.

This research was supported by the NRF Grant No. 2012R1A1A2003678 (H.H.), 2013R1A6A3A03028463(J.U.), and 2013R1A1A2A10009722(H.P.).
[1] S. N. Dorogovtsev, A. V. Goltsev, and J. F. F. Mendes, Rev. Mod. Phys. 80, 1275 (2008); see also the references therein.

[2] J. D. Noh and H. Park, Phys. Rev. E 79, 056115 (2009).

[3] M. Boguñá, C. Castellano, and R. Pastor-Satorras, Phys. Rev. E 79, 036110 (2009).

[4] S. H. Lee, M. Ha, H. Jeong, J. D. Noh, and H. Park,
Phys. Rev. E 80, 051127 (2009).

[5] T. Ichinomiya, Phys. Rev. E 70, 026116 (2004).

[6] D.-S. Lee, Phys. Rev. E 72, 026208 (2005); E. Oh, D.-S. Lee, B. Kahng, and D. Kim, ibid. 75, 011104 (2007).

[7] J. G. Restrepo, E. Ott, and B. R. Hunt, Phys. Rev. E 71, 036151 (2005).

[8] H. Hong, H. Park, and L.-H. Tang, Phys. Rev. E 76, 
$066104(2007)$

[9] H. Hong, M. Y. Choi, and B. J. Kim, Phys. Rev. E 65, 026139 (2002).

[10] J. Gómez-Gardeñes, Y. Moreno, and A. Arenas, Phys. Rev. E 75, 066106 (2007); Phys. Rev. Lett. 98, 034101 (2007).

[11] H. Hong, J. Um, and H. Park, Phys. Rev. E 87, 042105 (2013).

[12] H. Hong, M. Ha, and H. Park, Phys. Rev. Lett 98, 258701 (2007).

[13] C. Castellano and R. Pastor-Satorras, Phys. Rev. Lett. 105, 218701 (2010).

[14] H. K. Lee, P.-S. Shim, and J. D. Noh, Phys. Rev. E 87, 062812 (2013).

[15] M. Boguñá, C. Castellano, and R. Pastor-Satorras, Phys. Rev. Lett. 111, 068701 (2013).

[16] H. K. Lee, P.-S. Shim, and J. D. Noh, arXiv:1309.5367
[17] P. Erdös and A. Rényi, Publicationes Mathematical Debrencen 6, 290 (1959).

[18] Y. Kuramoto, in Proceedings of the International Symposium on Mathematical Problems in Theoretical Physics, edited by H. Araki (Springer-Verlag, New York, 1975); Chemical Oscillators, Waves, and Turbulence (SpringerVerlag, Berlin, 1984).

[19] D. Pazó, Phys. Rev. E 72, 046211 (2005).

[20] J. D. Crawford, J. Stat. Phys. 74, 1047 (1994).

[21] E. A. Martens, E. Barreto, S. H. Strogatz, E. Ott, P. So, and T. M. Antonsen, Phys. Rev. E 79, 026204 (2009).

[22] H. Hong, H. Park, and M. Y. Choi, Phys. Rev. E 72, 036217 (2005).

[23] H. Hong, H. Chaté, H. Park, and L.-H. Tang, Phys. Rev. Lett. 99, 184101 (2007). 